\title{
Suggestions for improving the design of clinical trials in multiple sclerosis-results of a systematic analysis of completed phase III trials
}

\author{
Sinje Gehr ${ }^{1}$ D $\cdot$ Thomas Kaiser $^{2} \cdot$ Reinhold Kreutz $^{1} \cdot$ Wolf-Dieter Ludwig $^{3} \cdot$ Friedemann Paul $^{1}$ (D)
}

Received: 8 August 2019 / Accepted: 18 October 2019 / Published online: 22 November 2019

(C) The Author(s) 2019

\begin{abstract}
This manuscript reviews the primary and secondary endpoints of pivotal phase III trials with immunomodulatory drugs in multiple sclerosis (MS). Considering the limitations of previous trial designs, we propose new standards for the planning of clinical trials, taking into account latest insights into MS pathophysiology and patient-relevant aspects. Using a systematic overview of published phase III (pivotal) trials performed as part of application for drug market approval, we evaluate the following characteristics: trial duration, number of trial participants, comparators, and endpoints (primary, secondary, magnetic resonance imaging outcome, and patient-reported outcomes). From a patient perspective, the primary and secondary endpoints of clinical trials are only partially relevant. High-quality trial data pertaining to efficacy and safety that stretch beyond the time frame of pivotal trials are almost non-existent. Understanding of long-term benefits and risks of disease-modifying MS therapy is largely lacking. Concrete proposals for the trial designs of relapsing (remitting) multiple sclerosis/clinically isolated syndrome, primary progressive multiple sclerosis, and secondary progressive multiple sclerosis (e.g., study duration, mechanism of action, and choice of endpoints) are presented based on the results of the systematic overview. Given the increasing number of available immunotherapies, the therapeutic strategy in MS has shifted from a mere "relapse-prevention" approach to a personalized provision of medical care as to the choice of the appropriate drugs and their sequential application over the course of the disease. This personalized provision takes patient preferences as well as disease-related factors into consideration such as objective clinical and radiographic findings but also very burdensome symptoms such as fatigue, depression, and cognitive impairment. Future trial designs in MS will have to assign higher relevance to these patient-reported outcomes and will also have to implement surrogate measures that can serve as predictive markers for individual treatment response to new and investigational immunotherapies. This is an indispensable prerequisite to maximize the benefit of individual patients when participating in clinical trials. Moreover, such appropriate trial designs and suitable enrolment criteria that correspond to the mode of action of the study drug will facilitate targeted prevention of adverse events, thus mitigating risks for individual study participants.
\end{abstract}

Keywords Multiple sclerosis · Clinical trial · Phase III · Patient-reported outcome measures (PROM) · Comparator · Patient preferences · Personalized provision of medical care · Predictive surrogate measures · Targeted prevention · Predictive preventive personalised medicine $\cdot$ Immunotherapy $\cdot$ Immunomodulatory drugs $\cdot$ Patient stratification $\cdot$ Patient benefits $\cdot$ Risk analysis $\cdot$ Mitigation $\cdot$ Relapse-prevention approach $\cdot$ Fatigue $\cdot$ Depression $\cdot$ Cognitive impairment $\cdot$ Criteria

Sinje Gehr

sinje.gehr@charite.de

1 Charité Universitätsmedizin Berlin, Charitéplatz 1, 10117 Berlin, Germany
2 Institut für Qualität und Wirtschaftlichkeit im Gesundheitswesen (Institute for Quality and Efficiency in Health Care) (IQWiG), Im Mediapark 8, 50670 Köln, Germany

3 Arzneimittelkommission der deutschen Ärzteschaft (Drug Commission of the German Medical Association), Herbert-Lewin-Platz 1, 10623 Berlin, Germany 


\section{Background}

Since the development of the first immunotherapy interferon beta-1b in 1995 (see Table 1), a number of immunomodulatory substances have been authorized for the diseasemodifying treatment of multiple sclerosis (MS), namely by reducing relapse rates $[13,25,58]$. The mechanisms of action have been fully elucidated for only few of these drugs. While a positive effect on the autoreactive, inflammatory immune response is considered proven, direct neuroprotective effects are unlikely.

All drugs licensed to date were tested in 1- to 2-year (rarely longer) pivotal trials, mostly against placebo [20], although more recently, active comparators have also begun to be applied. From the patient's point of view, some of the primary and secondary endpoints of these studies have limited relevance [27, 65]. Moreover, methodologically sound data on these drugs' efficacy and safety (or detrimental effects), beyond the duration of these trials, are practically non-existent. The little data covering 3 years or more of application mostly derive from "extension studies" to initial phase III studies or from registers such as "MSBase" [36]. Specialized statistical analyses are applied to compensate for the poor methodological quality of "observational studies" in order to gain insight into the efficacy of immunomodulatory treatments (including compared with each other). However, the "real-world" data gathered in registers are generally not suited for such analyses [31]. Overall, these factors suggest a general approach to designing clinical MS trials that leaves room for improvement and which has hampered our understanding of the long-term benefits and risks of diseasemodifying MS treatment. However, these deepened insights are urgently needed to enable neurologists to proceed from a mere "relapse-preventative" strategy when prescribing immunotherapies towards provision of personalized medical services that take the multiple facets of the disease and patient preferences into consideration [22, 45] and also adopts the aim of targeted prevention of adverse events.

\section{Investigative goal}

The goal of this study is, firstly, to set out an overview of the primary and secondary endpoints of pivotal phase III trials in MS. Secondly, based on this summary, as well as our analysis of the shortcomings of clinical trial design to date, we propose a number of suggestions for improvement. Here, we also draw on the latest insights into MS pathophysiology, as well as aspects relevant for patients, particularly the implementation of "patient-reported outcome measures" (PROM). Moreover, we describe the ongoing, significant demand for trials with therapeutic agents that modify disease progression, for which there have been too few controlled studies to date.

\section{Materials and methods}

Our research of the available literature yielded a systematic overview of published pivotal phase III MS trials performed to provide evidence for drug marketing approval (so-called pivotal trials). We took as a starting point an assessment of 21 randomized, controlled phase III trials on relapsing-remitting multiple sclerosis (RRMS) presented in Torkildsen et al. [74]. As all of the latter were completed prior to May 21, 2015, we augmented them with our own research into the literature, focusing on further completed and published phase III MS trials (inclusion criteria), as well as extending analysis of all the included trials to the disease courses relapsing multiple sclerosis (RMS), primary progressive MS (PPMS), secondary progressive MS (SPMS), and clinically isolated syndrome (CIS). Drugs not approved for the market despite phase III trial were not included (exclusion criteria). The literature was searched using PubMed, as well as the European public assessment reports (EPAR) of the European Medicines Agency (EMA) and the dossier assessments of early benefit assessments conducted by the German Institute for Quality and Efficiency in Health Care (IQWiG). The PubMed search was conducted using the keywords Multiple Sclerosis, Phase 3, trial, with last access on 2-15-2019.

The following characteristics of the phase III trials were analyzed: trial duration, sample size, comparator drugs (primary, secondary, MRI (magnetic resonance imaging) outcomes, as well as patient-reported outcome measures (PROM)).

\section{Results}

Table 1 of the Appendix summarizes the characteristics of the 29 assessed pivotal phase III trials. Below we describe the key results of our investigation of the trials for the disease courses RRMS, RMS, SPMS, PPMS, and CIS.

\section{Trial duration}

The analysis showed that the phase III RRMS trials conducted since the 1990s had a duration of approximately 2 years, with some few exceptions (e.g., EVIDENCE trial, interferon beta-1a, 1-year duration). For RMS, the trial duration was also generally 2 years. Exceptions here were 


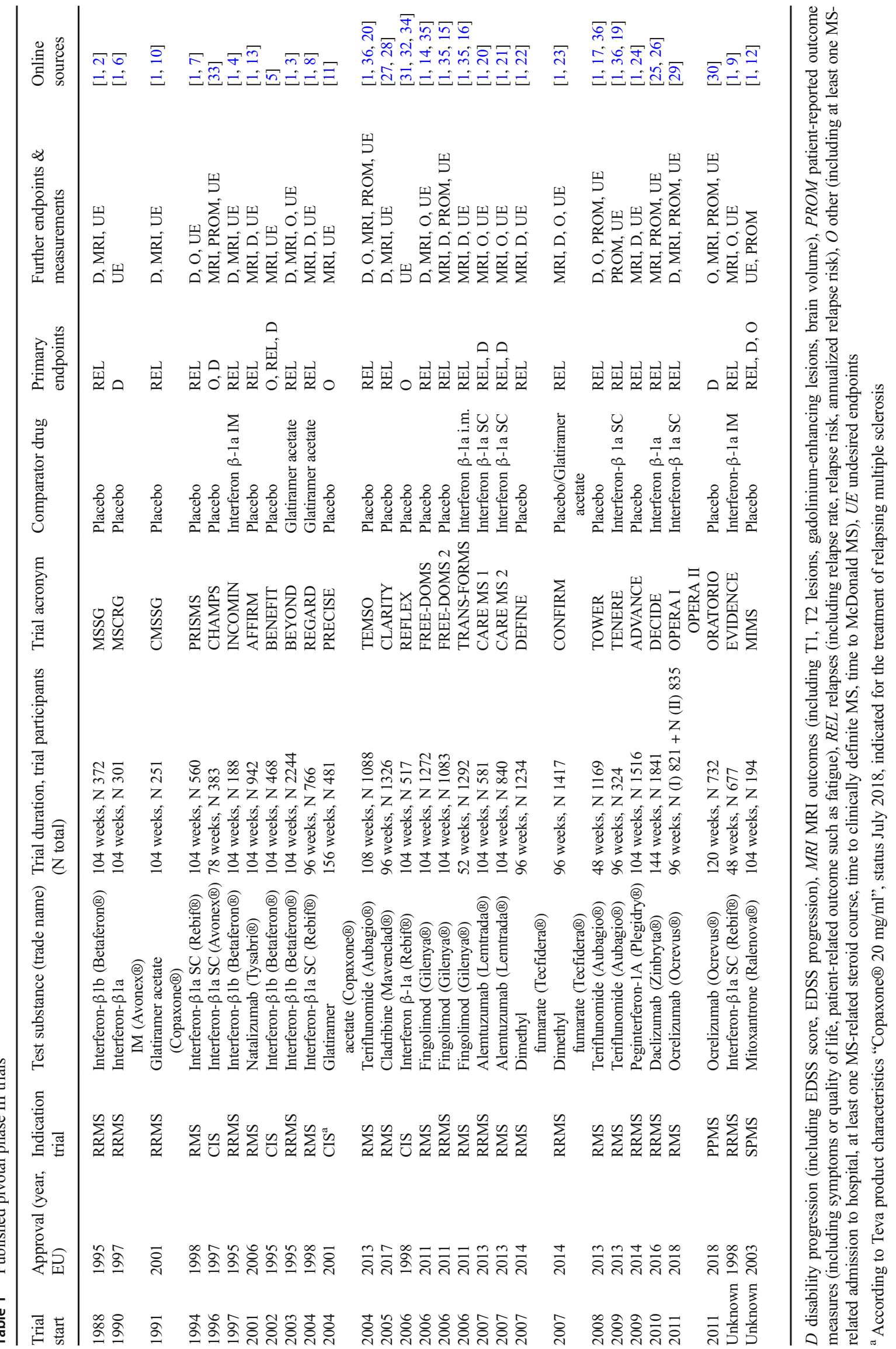


the 1-year TRANSFORMS trial (fingolimod, approved 2011) and the TOWER trial (teriflunomide, approved 2013), which had a variable duration, but was already completed 48 weeks after inclusion of the last patient. Alone, the PRECISE trial (glatiramer acetate for CIS, approved 2001) had a study duration of 3 years. Only recently have trials longer than 2 years been carried out, including the DECIDE trial (daclizumab for RRMS, 144 weeks, approved 2016, market withdrawal 2018) and the ORATORIO trial in PPMS (ocrelizumab, 120 weeks, approved 2018).

\section{Number of participants in MS pivotal trials}

In recent years, the number of trial participants has increased significantly. While the first pivotal interferon beta 1b trial MSSG only included 372 patients, the DECIDE trial (daclizumab) recruited 1841 patients. One of the reasons for this is that relapse rates have decreased significantly over the last 20 years, for instance because many patients are already being treated with immunomodulatory agents and therefore patients with milder disease course are more likely to be recruited for drug trials. Thus, today significantly higher case numbers are needed to reach statistical significance using annual relapse rate as primary endpoint, with absolute differences between investigational medicinal product (IMP) and comparator drug sometimes averaging just $<0.2$ relapses per year. Miniscule effects that only reach statistical significance by inflation of sample size suggests that such trial results are of questionable clinical relevance. However, it should be noted that one advantage of larger sample sizes is a greater chance of detecting rare side effects.

\section{Comparator drugs}

The earliest RRMS trials tested the IMP against placebo as no other immunomodulatory agents had yet been developed. However, more recently, trials are increasingly carried out against active comparators, such as against interferon beta$1 \mathrm{a}$ in the RRMS trials CARE MS-1 (alemtuzumab), DECIDE (daclizumab), and EVIDENCE (SC vs. IM interferon beta-1a). Glatiramer acetate was used as comparator drug in the BEYOND trial (interferon beta-1b). In RMS, seven placebo-controlled trials and four trials (TRANSFORMS (fingolimod), CARE MS-2 (alemtuzumab), TENERE (teriflunomide), and OPERA I + II (ocrelizumab)) with interferon beta-1a as active comparator were carried out. In the RMS trial REGARD (interferon beta-1a) glatiramer acetate served as active comparator. The cytotoxic agent mitoxantrone for SPMS was tested against placebo in the MIMS trial, as was the monoclonal antibody ocrelizumab for PPMS in the ORATORIO trial. All four trials in CIS were also placebo-controlled: CHAMPS (interferon- $\beta 1 \mathrm{a}$ ), BENEFIT (interferon- $\beta 1 \mathrm{~b}$ ), PRECISE (glatiramer acetate), and REFLEX (interferon- $\beta 1 \mathrm{a})$.

\section{Endpoints}

In RRMS trials, relapse rate was most frequently selected as primary endpoint. Disability progression, measured according to the "Expanded Disability Status Scale (EDSS)" for the quantification of neurological disability and confirmed after 12 or 24 weeks, was selected as primary endpoint in two trials (interferon- $\beta 1 \mathrm{a}, \mathrm{MSCRG}$, and alemtuzumab, CARE MS 1), but served only as secondary endpoint in most. Apart from adverse events, key secondary or explorative endpoints included MRI endpoints, such as number and volume of gadolinium-enhancing lesions and T2-hyperintense or T1-hypointense lesions in cranial MRI and, most recently, also the progression of cerebral atrophy [61]. For the 12 RMS trials, only relapse rate was selected as primary endpoint, albeit in the case of alemtuzumab (CARE MS 1 trial) in combination with disability progression. The pattern was similar for clinically isolated syndrome (CIS): the primary endpoints of the PRECISE trial (glatiramer acetate) were the rate of conversion to clinically definite MS as defined by a second clinical event, while the BENEFIT trial (interferon- $\beta$ 1b) measured conversion to both clinically definite MS and McDonald MS, as well as the annual relapse rate and the degree of disability. Apart from the primary endpoint ("disability progression confirmed at 12 weeks"), the PPMS trial on ocrelizumab (ORATORIO trial) also investigated secondary endpoints such as "disability progression confirmed at 24 weeks", MRI endpoints, as well as a patient-reported outcome (quality of life according to the SF-36 (Short Form (36) Health Survey)).

\section{Patient-reported outcome measures (PROM)}

In many cases, patients and physicians differ in the importance ascribed to particular symptoms and consequences of the disease [27, 65]. In general, patients tend to focus far more on disability progression impacting quality of life, rather than disease progression as measured by anatomical, biological, and clinical data. As such, patients generally understand disease progression as the worsening of symptoms, with fatigue, depression, cognitive impairment, pain, spasticity, sleep disturbance, loss of visual functioning, and mobility among those considered most burdensome $[16,17,24,27,57,60,63,65$, $77,78,80]$. Many of these symptoms can be easily quantified using internationally established and validated patient questionnaires. 
Virtually, no drug approval trial has systematically investigated PROM. Where investigated, the focus is on fatigue, which is considered by many MS patients to be one of the most troubling symptoms [79]. Here, two examples are the TENERE and TEMSO trials (both teriflunomide, RMS), which investigated fatigue as secondary endpoint using the "Fatigue Impact Scale (FIS)". In the ocrelizumab trials (OPERA I, OPERA II, ORATORIO), health-related quality of life (HRQoL) was measured using the established, generic survey SF-36, which comprises the section vitality, physical functioning, bodily pain, general health perceptions, physical role functioning, emotional role functioning, social role functioning, and mental health. Nevertheless, measuring HRQoL is far from standard in clinical MS trials, as evidenced by the CLARITY trial for the oral drug cladribine [34], which did not include any PROM parameters [21]. Overall, PROM are still investigated less frequently as primary or secondary endpoints than relapse rate, disease progression, or MRI parameters.

\section{Discussion}

\section{Deficits in the design of phase III trials to date}

Systematic analysis of the phase III trials included in this overview showed that the approval of new substances for the treatment of multiple sclerosis were as a rule randomized, controlled studies of at least 1 year and each included several hundred, sometimes over 1000, patients. This, in principle, suggests that an established approach to designing MS clinical trials exists to a greater extent than in other neurological disorders. However, on closer inspection, it becomes clear that our approach to MS clinical trial design urgently needs to redirect focus towards patient needs, as opposed to biological indicators and surrogate measures of dubious clinical importance.

In the EMA's "Guideline on clinical investigation of medicinal products for the treatment of Multiple Sclerosis" [30], relapse rate and disability progression are singled out as the most important primary endpoints. The guideline distinguishes between the "accumulation of disability" in terms of relapse rate in RMS and disability progression in SPMS or PPMS in phase III trials, with clinically measured prevention or delay of disability progression recommended as primary endpoint for SPMS and PPMS. For patients with RRMS or SPMS with relapsing MS (RMS), both relapse rate and the time to relapse are accepted as primary outcomes. Relapse rate, or that is, the proportion of relapse-free patients along with the progression of disability should, in addition to MRI outcomes, be investigated as secondary endpoints, insofar as they have not already been examined as primary endpoints. Furthermore, the EMA guideline calls for more emphasis on PROM, as symptoms such as subjective visual function, pain, bladder control, depression, sleep disorder, fatigue, and cognitive dysfunction are enormously important for quality of life and are considered more crucial by some patients than purely somatic outcomes $[26,65]$.

Analysis of the phase III trials to date highlights significant deficits in pivotal MS trials. These include a treatment duration that is often too short, discrepancies between the hypothesized mechanism of action and inclusion criteria (inclusion of patients with little disease activity or very long disease duration in trials with substances that have strong anti-inflammatory effect), premature confirmation of disease progression (already after 12 weeks), or the lack of relevant PROM. The attempt to obtain statistical significance with high patient numbers despite often only minimal absolute differences in the relapse rate between IMP and comparator drug are both of questionable clinical relevance and of dubious cost-effectiveness (insofar as these resources are then not available for other trials).

Investigating outcomes that are particularly important to patients has been the exception in drug approval trials to date and where the case, they only serve as secondary or explorative endpoints. These approaches are methodologically inadequate to some extent, for example because the PROM explorative endpoints were usually not surveyed using validated measurement instruments (e.g., measurement of fatigue in the TRANSFORMS trial on fingolimod with the unvalidated questionnaire "draft 39item version of the U-FIS”) [35].

\section{Suggestions for improving phase III MS trial design}

The hypothesized mechanism of action should be clearly described at the beginning of the trial and should be taken into consideration when designing the study. For immunomodulatory drugs intended for treatment in (highly) active disease stages (e.g., natalizumab, ocrelizumab), this would mean that relapse rate could continue to serve as endpoint. However, aspects such as the severity of the relapse or the functional disability and the remission should also be taken into account. To establish the added benefit of a new drug, a clinically significant effect on functionally debilitating relapses (e.g., visual functioning, mobility, physical strength) should be demonstrated. As the timing of relapses is difficult to predict, the duration of observation in trials that take relapse rate as endpoint should be at least 2 years.

For substances with a hypothesized effect on disability progression, the observation period should be of suitable 
duration (at least 3 years, ideally up to 5 years). Currently, disability progression is commonly established after only 12 or 24 weeks. However, recovery from relapse can take up to 6 or even 12 months, and temporary disability changes stemming from previous relapses can lead to overestimation of long-term disability progression. Consequently, disability progression should only be confirmed after 12 months, which reduces confounding effect of incomplete recovery from recent relapses [42].

The inclusion criteria for the trial subjects should take into account the expected effect of the active substance and be compatible with the primary endpoint. For example, validation of a drug with hypothesized effect on disability progression should include patients whose progression is confirmed prior to inclusion in the trial. Otherwise, the danger exists of including significant numbers of "stable" patients or those with only slow progression. In such cases, the drug being tested might indeed have an effect in the subpopulation, but this might not be detected in the sample due to the mild natural history of the cohort (false negative result for the subpopulation in question). Conversely, a "positive" effect could be the result of the actual subpopulation of interest, but be mistakenly extended to include patients with mild natural history (false positive result for the subpopulation with mild natural history, see ORATORIO trial, ocrelizumab).

For drugs with strong anti-inflammatory effect, focus should be placed on including patients in early disease phases with higher disease activity, instead of - as was most recently the case in the CLARITY trial-a very broad enrolment of the population, including patients with low disease activity and extremely long disease duration. By ensuring the inclusion criteria is compatible with the hypothesized mode of action, it might be possible to achieve clinically relevant results with smaller case numbers, thereby sparing patients the risks involved in testing new immunotherapies. The subjects should also cover a wide age range (up to 60 or 65 years, as opposed to the currently usual age limit of 55) and age effects should be investigated, as a recent meta-analysis demonstrated that age can affect the efficacy of an immunomodulatory therapy [82]. Apart from EDSS, it is imperative that a functional test of lowcontrast sensitivity (e.g., low contrast letter acuity using Sloan charts) and mobility (e.g., 6-min walk test) be performed to quantify disability, as vision and mobility are the most important bodily symptoms from a patient point of view [27].

The EMA guideline [30] recommends evaluating health-related quality of life, although lack of data precludes recommending any specific instruments. Indeed, using established assessment instruments to quantify health-related quality of life, as well as fatigue and cognition, should become standard practice. Tools for measuring quality of life include MSQOL 54 (Multiple Sclerosis Quality of Life-54) [38], HAQUAMS (Hamburg Quality of Life Questionnaire in Multiple Sclerosis) [66], MSQLI (Multiple Sclerosis Quality of Life Inventory) [39], or the recently developed NeuroQoL [51]. Fatigue and cognition can be measured using the Fatigue Severity Scale (FSS) [37, 46] and BICAMS (Brief International Cognitive Assessment for Multiple Sclerosis) [32, 48], respectively. A simple further screening instrument for cognition is the SDMT (Symbol Digit Modalities Test) [75]. The effect of limited vision and mobility on quality of life should be quantified using the established measurement instrument NEI-VFQ25 (National Eye Institute Visual Functioning Questionnaire 25) [40] or the MSWS-12 [23], as applies.

Cerebral or spinal MRI parameters can serve as secondary or explorative outcomes (e.g., T2 lesions, spinal cord atrophy [2, 83]); however, the repeated application of gadolinium-based MRI contrast agents should be avoided due to safety concerns [14, 67]. Moreover, brain atrophy measurements, although technically feasible in a clinical study with rigorous standardization of assessments, are not recommended as they are not yet transferable to gauge prediction and monitoring of disease course in individual patients, thus currently not supporting personalization of medical services $[3,28,61,62,81]$. The same applies to other advanced imaging modalities such as diffusion tensor imaging, ultrahigh field MRI and others $[47,56,59,64$, $69,70,72]$. Most recently, the use of retinal optical coherence tomography (OCT) for the quantification of axonal and neuronal damage caused by MS is increasing [4, 10, $50,52-54,84]$. This technique has been occasionally used as outcome measure in clinical trials and might serve as predictive diagnostics for disease course and response to immunotherapy both in trial cohorts and individual subjects in the future. A further suitable secondary or explorative outcome that might be established in the near future both for clinical studies and individualized prediction is the identification of neurofilaments in serum as surrogate marker of axonal damage in the CNS $[1,6,7,12,43,49$, 73, 76].

Exclusively placebo-controlled trials with a duration of more than 6 months that test disease-modifying drugs in RRMS are ethically problematic. Moreover and importantly, the advantages and disadvantages of individual treatment options cannot be identified by means of placebo- 
controlled studies. When selecting appropriate active comparators, care should be taken that the trial's inclusion criteria reflect both the study population and the active comparator's approval status. In trials with a PPMS population, the lack of approved drugs (with the exception of ocrelizumab) justifies the use placebo-controlled trials. Ocrelizumab, which was recently approved, likely only benefits a small subgroup of younger PPMS patients (up to 45 years) with short disease duration (up to 15 years) and disease activity in MRI (new T2 lesions, gadoliniumenhancing lesions), and trialing against this substance in other PPMS populations (older patients, longer disease duration, no MRI activity) makes little sense and is moreover not ethically acceptable.

Overall, the high demand of patients for studies in progressive MS (SPMS, PPMS) continues. A significant number of drugs, including some approved for treatment of RRMS, were unsuccessfully tested in patients with progressive MS. The reasons for this are manifold and include an incomplete understanding of the pathophysiology of progression, an insufficiently detailed grasp of the mechanism of action of the drug, and the shortcomings of the applied outcomes (such as the EDSS with high inter-rater variability and disproportionate weighting allocated to lower extremity functioning, or more generally, the ability to walk).

The suggestions for improvement presented here build to some extent on those recently published by Ontaneda et al. ([55], see Text Box 5b). Importantly, clinical researchers planning a trial should make use of the EMA's advice service, and ideally also involve the European HTA (Health Technology Assessment) institutions [29], of which the Federal Joint Committee (GBA) is the primary body in Germany [33]. The aim of the consultation process should be ensuring that the trial is designed to not only meet the requirements necessary for market approval, but also to inform treatment decisions and to perform an assessment of the added benefit of the new drug compared to standard treatment. The results of the consultation should be published to ensure transparency.

Pharmaceutical companies in Germany sometimes complain that the recommendations of drug approval agencies and HTA institutions (IQWiG and GBA) diverge to some extent and that data from a single phase III trial is interpreted differently by the EMA, on the one hand, and the committees participating in the early benefit assessment (IQWiG and G-BA), on the other. However, this perspective does not take into account the fact that the questions posed by the approval agencies ("Does the new substance have a positive benefit-risk balance?") and the benefit assessment ("Does the new substance have an added benefit compared to a "standard therapy'?") are very different. By necessity, this leads to diverging demands on the trial design in the first instance, and ultimately leads to different interpretation of the obtained results in the final instance.

Despite our criticism of MS studies to date, we are aware that planning a clinical trial and improving quality and patient focus as discussed above also (have to) take into account a wider health policy and regulatory framework, as well as financial considerations. This complicates and could even hobble design of a study that focuses exclusively on achieving scientific insights into MS.

\section{Expert recommendations}

Fortunately, the therapeutic landscape for people with MS has significantly broadened over the past 15 years. In parallel to the increasing number of available immunotherapies, treatment strategies in MS have shifted from a mere "relapse-prevention" approach to a personalized provision of medical care as to the choice of the appropriate drugs and their sequential application over the course of the disease. This personalized provision should take patient preferences as well as disease-related factors into consideration such as objective clinical and radiographic findings but also very burdensome symptoms such as fatigue, depression, and cognitive impairment. This change in perspective on what physicians want to accomplish for their patients has not only been endorsed by clinicians and researchers but has also been adopted by regulatory bodies such as EMA. Therefore, future trial designs in MS should assign higher relevance to these patient-reported outcomes and should aim at implementing measures that can serve as predictive markers for individual treatment response to new and investigational immunotherapies. This is an indispensable prerequisite to maximize the benefit of individual patients when participating in clinical trials and when starting on an immunotherapy post approval. Moreover, such appropriate trial designs and suitable enrolment criteria that correspond to the mode of action of the study drug will facilitate targeted prevention of adverse events, thus mitigating risks for individual study participants. Finally, personalized provision of medical services prior to enrolment into clinical trials must encompass utmost accuracy when diagnosing MS and ruling out relevant differential diagnoses given that newer and highly efficacious immunotherapies for MS might cause harm in other MS mimics such as neuromyelitis optica spectrum disorders and many others $[5,8,9,11$, $15,18,19,41,44,53,68,71]$. 
5a Text box "Suggestions for improving the design of (R) $\mathrm{RMS}^{1} / \mathrm{CIS}$ trials"

Duration: Not less than 2 years, active comparator

Outcomes: Relapse rate, only functionally debilitating (relapse involving EDSS worsening of at least 1 point) relapses with full or partial recovery should be included

Disability progression: in RRMS trials involving patients with short disease duration, it makes little sense to investigate after 12 or 24 weeks; instead the number of functionally debilitating relapses and their remission should be examined; comparing disability at the end of a 2-year trial compared to baseline recommended

Additional for CIS: Time to conversion to CDMS (Clinically Definite Multiple Sclerosis)

Further outcomes/measurement instruments:

- General: EDSS, MSFC (Multiple Sclerosis Functional Composite)

- Vision: Snellen Visual Acuity Test and LCLA (Low Contrast Letter Acuity), e.g., using Sloan charts)

- Mobility: 6-min walk test

- Cognition: BICAMS or at a minimum SDMT

- PROM: Fatigue (FSMC (Fatigue Scale for Motor and Cognitive Functions)) or FSS), depression BDI-II (Beck Depression Inventory II); general quality of life: HAQUAMS (Hamburg Quality of Life Questionnaire in Multiple Sclerosis), SF36 or MSQoL; vision-related quality of life: NEI-VFQ25; mobility-related quality of life: MSWS-12 (Multiple Sclerosis Walking Scale); sleep-related quality of life: PSQI (Pittsburgh Sleep Quality Inventory); pain-related quality of life: Brief Pain Inventory (BPI)

- MRI: Repeated administration of gadolinium-based contrast agent to be avoided, T2 lesions recommended, brain atrophy not recommended as clinical value unclear, spinal cord atrophy as potentially promising imaging marker

- OCT (Optical Coherence Tomography) with GCIPL (Ganglion Cell-inner Plexiform Layer) and RNFL (Retinal Nerve Fiber Layer)

5b Text box "Suggestions for improving the design of PPMS/SPMS ${ }^{2}$ trials"

Prerequisite: Inclusion only of patients with proven clinical progression PRIOR to inclusion (e.g., at least 1 EDSS point in prior 1-2 years)

Duration not less than 3 years, preferably up to 5 years, placebo may need to be justified

Endpoints: Confirmed disability progression after 12 months

Further endpoints/measurement instruments:

- General: EDSS, MSFC (Multiple Sclerosis Functional Composite)

- Vision: Visual Acuity Test and LCLA (Low Contrast Letter Acuity), e.g., using Sloan charts)

- Mobility: 6-min walk test

- Cognition: BICAMS or at a minimum SDMT

- PROM: Fatigue (FSMC (Fatigue Scale for Motor and Cognitive Functions)) or FSS), depression BDI-II (Beck Depression Inventory II); general quality of life: HAQUAMS (Hamburg Quality of Life Questionnaire in Multiple Sclerosis), SF36, MSQoL or Neuro-QoL; vision-related quality of life: NEI-VFQ25; mobility-related quality of life: MSWS-12 (Multiple Sclerosis Walking Scale); sleep-related quality of life: PSQI (Pittsburgh Sleep Quality Inventory); pain-related quality of life: Brief Pain Inventory (BPI)

- MRI: Repeated administration of gadolinium-based contrast agent to be avoided, T2 lesions recommended, brain atrophy not recommended as clinical value unclear, spinal cord atrophy as potentially promising imaging marker

- OCT (optical coherence tomography) with GCIPL (ganglion cell-inner plexiform layer), and RNFL (retinal nerve fiber layer)

\footnotetext{
${ }^{1}$ RMS also includes patients with SPMS and relapse activity

${ }^{2}$ Particularly patients without relapse activity
}

Abbreviations $A M N O G$, The Act on the Reform of the Market for Medical Products; BDI-II, Beck Depression Inventory II; BICAMS, Brief International Cognitive Assessment for Multiple Sclerosis; $B P I$, Brief Pain Inventory; $C D M S$, Clinically definite multiple sclerosis; $C I S$, Clinically isolated syndrome; EDSS, Expanded Disability Status Scale; EMA, European Medicines Agency; EPAR, European Public Assessment Reports; FIS, Fatigue Impact ScalevFSMC, Fatigue Scale for Motor and Cognitive Functions; FSS, Fatigue Severity Scale; $G-B A$, Federal Joint Committee; GCIPL, plexiform layer; HAQUAMS, Hamburg Quality of Life Questionnaire in Multiple Sclerosis; HTA, Health technology assessment; IQWiG, German Institute for Quality and Efficiency in Health Care; LCLA, Low Contrast Letter Acuity; MRI, Magnetic resonance imaging; $M S$, Multiple sclerosis; $M S F C$, Multiple Sclerosis Functional Composite; MSWS-12, Multiple Sclerosis Walking Scale; MSQLI, Multiple Sclerosis Quality of Life Inventory; MSQOL-54, Multiple Sclerosis Quality of Life-54; NEI-VFQ25, National Eye Institute Visual Functioning Questionnaire 25; Neuro-QoL, Quality of Life in Neurological Disorders; OCT, Optical coherence tomography; PPMS, Primary progressive multiple sclerosis; $P R O M$, Patient-reported outcome measure; PSQI, Pittsburgh Sleep Quality Inventory; $R M S$, Relapsing multiple sclerosis; $R N F L$, Retinal nerve fiber layer; $R R M S$, Relapsingremitting multiple sclerosis; $S D M T$, Symbol Digit Modalities Test; SF36, 36-Item Short Form Survey; SPMS, Secondary progressive multiple sclerosis

\section{Compliance with ethical standards}

Conflicts of interests Sinje Gehr, Thomas Kaiser, and Wolf-Dieter Ludwig declare that they have no competing interest. Friedemann Paul received lectures fee/research support from Bayer/Novartis/Biogen Idec/TEVA/Sanovi-Aventis-Genzyme/Merck Serono, Alexion/Chugai/ Medimmune/Roche/Chugai/Guthy Jackson Foundation. Furthermore, Friedemann Paul took part in the advisory boards of Novartis and Medimmune. Reinhold Kreutz declares that he consulted Bayer Pharma, Berlin-Chemie, Daiichi Sankyo, Ferrer, Sanofi, Servier. Additionally, he did research for Bayer Pharma.

Ethical approval This article does not contain any studies with human participants or animals performed by any of the authors. The consent of the publication is not applicable. Ethical approval is not applicable.

Open Access This article is distributed under the terms of the Creative Commons Attribution 4.0 International License (http:// creativecommons.org/licenses/by/4.0/), which permits unrestricted use, distribution, and reproduction in any medium, provided you give appropriate credit to the original author(s) and the source, provide a link to the Creative Commons license, and indicate if changes were made.

\section{References}

1. Akgun K, Kretschmann N, Haase R, et al. Profiling individual clinical responses by high-frequency serum neurofilament assessment in MS. Neurol Neuroimmunol Neuroinflamm. 2019;6:e555.

2. Alcaide-Leon P, Cybulsky K, Sankar S, et al. Quantitative spinal cord MRI in radiologically isolated syndrome. Neurol Neuroimmunol Neuroinflamm. 2018;5:e436.

3. Amiri H, De Sitter A, Bendfeldt K, et al. Urgent challenges in quantification and interpretation of brain grey matter atrophy in individual MS patients using MRI. Neuroimage Clin. 2018;19: 466-75.

4. Ayadi N, Dorr J, Motamedi S, et al. Temporal visual resolution and disease severity in MS. Neurol Neuroimmunol Neuroinflamm. 2018;5:e492. 
5. Beekman J, Keisler A, Pedraza O, et al. Neuromyelitis optica spectrum disorder: Patient experience and quality of life. Neurol Neuroimmunol Neuroinflamm. 2019;6:e580.

6. Bergman J, Dring A, Zetterberg H, et al. Neurofilament light in $\mathrm{CSF}$ and serum is a sensitive marker for axonal white matter injury in MS. Neurol Neuroimmunol Neuroinflamm. 2016;3:e271.

7. Bjornevik K, Munger KL, Cortese M et al. Serum Neurofilament Light Chain Levels in Patients With Presymptomatic Multiple Sclerosis. JAMA Neurol. 2019. https://doi.org/10.1001/ jamaneurol.2019.3238.

8. Blaabjerg M, Ruprecht K, Sinnecker T, et al. Widespread inflammation in CLIPPERS syndrome indicated by autopsy and ultrahigh-field 7T MRI. Neurol Neuroimmunol Neuroinflamm. 2016;3:e226.

9. Borisow N, Hellwig K, Paul F. Neuromyelitis optica spectrum disorders and pregnancy: relapse-preventive measures and personalized treatment strategies. EPMA J. 2018;9:249-56.

10. Cellerino M, Cordano C, Boffa G, et al. Relationship between retinal inner nuclear layer, age, and disease activity in progressive MS. Neurol Neuroimmunol Neuroinflamm. 2019. https://doi.org/ 10.1212/NXI.0000000000000596.

11. Cook LJ, Rose JW, Alvey JS et al. Collaborative International Research in Clinical and Longitudinal Experience Study in NMOSD. Neurol Neuroimmunol Neuroinflamm. 2019;6:e583

12. Dalmau J. Neurofilament light chain levels in MS: At the doorstep of clinical application. Neurol Neuroimmunol Neuroinflamm. 2019. https://doi.org/10.1212/NXI.0000000000000601.

13. Dorr J, Paul F. The transition from first-line to second-line therapy in multiple sclerosis. Curr Treat Options Neurol. 2015;17:354.

14. El-Khatib AH, Radbruch H, Trog S, et al. Gadolinium in human brain sections and colocalization with other elements. Neurol Neuroimmunol Neuroinflamm. 2019;6:e515.

15. Filippi M, Preziosa P, Banwell BL, Barkhof F, Ciccarelli O, de Stefano N, et al. Assessment of lesions on magnetic resonance imaging in multiple sclerosis: practical guidelines. Brain. 2019;142:1858-75.

16. Finke C, Schlichting J, Papazoglou S, et al. Altered basal ganglia functional connectivity in multiple sclerosis patients with fatigue. Mult Scler. 2015;21:925-34.

17. Gaede G, Tiede M, Lorenz I, et al. Safety and preliminary efficacy of deep transcranial magnetic stimulation in MS-related fatigue. Neurol Neuroimmunol Neuroinflamm. 2018;5:e423.

18. Gahlen A, Trampe AK, Haupeltshofer S, et al. Aquaporin-4 antibodies in patients treated with natalizumab for suspected MS. Neurol Neuroimmunol Neuroinflamm. 2017;4:e363.

19. Geraldes R, Ciccarelli O, Barkhof F, et al. The current role of MRI in differentiating multiple sclerosis from its imaging mimics. Nat Rev Neurol. 2018;14:199-213.

20. Gerardi C, Bertele V, Rossi S, et al. Preapproval and postapproval evidence on drugs for multiple sclerosis. Neurology. 2018;90: 964-73.

21. Gesundheitswesen IFQUWI (2018) [A17-62] Cladribin (multiple Sklerose) - Nutzenbewertung gemäß § 35a SGBV.

22. Golubnitschaja O, Baban B, Boniolo G, et al. Medicine in the early twenty-first century: paradigm and anticipation - EPMA position paper 2016. EPMA J. 2016;7:23.

23. Grobelny A, Behrens JR, Mertens S, et al. Maximum walking speed in multiple sclerosis assessed with visual perceptive computing. PLoS One. 2017;12:e0189281.

24. Hasselmann H, Bellmann-Strobl J, Ricken R, et al. Characterizing the phenotype of multiple sclerosis-associated depression in comparison with idiopathic major depression. Mult Scler. 2016;22: 1476-84.

25. Havla J, Warnke C, Derfuss T, Kappos L, Hartung HP, Hohlfeld R. Interdisciplinary Risk Management in the Treatment of Multiple Sclerosis. Dtsch Arztebl Int. 2016;113:879-86.
26. Heesen C, Bohm J, Reich C, et al. Patient perception of bodily functions in multiple sclerosis: gait and visual function are the most valuable. Mult Scler. 2008;14:988-91.

27. Heesen C, Haase R, Melzig S, Poettgen J, Berghoff M, Paul F, et al. Perceptions on the value of bodily functions in multiple sclerosis. Acta Neurol Scand. 2018;137:356-62.

28. Hemond CC, Healy BC, Tauhid S, et al. MRI phenotypes in MS: Longitudinal changes and miRNA signatures. Neurol Neuroimmunol Neuroinflamm. 2019;6:e530.

29. http://www.ema.europa.eu/docs/en_GB/document_library/ Regulatory_and_procedural_guideline/2017/07/Wc500230375. pdf. Accessed 10 Sept 2019.

30. http://www.ema.europa.eu/docs/en_gb/document_library/ scientific_guideline/2015/03/wc500185161.pdf. Accessed 10 Sept 2019.

31. https://www.aerzteblatt.de/archiv/188027/Patientenregister-fuerdie-Nutzenbewertung-Kein-Ersatz-fuer-randomisierte-Studien. Accessed 10 Sept 2019.

32. https://www.bicams.net/. Accessed 10 Sept 2019.

33. https://www.g-ba.de/Institution/Presse/Pressemitteilungen/695/. Accessed 10 Sept 2019.

34. https://www.iqwig.de/De/Projekte-Ergebnisse/Projekte/ Arzneimittelbewertung/2017/A17-62-Cladribin-MultipleSklerose-Nutzenbewertung-Gemaess-35a-Sgbv.8401.html. Accessed 10 Sept 2019.

35. https://www.iqwig.de/download/A14-21_Fingolimod-NeuesAnwendungsgebiet_Nutzenbewertung-35a-Sgb-V.pdf. Accessed 10 Sept 2019.

36. https://www.msbase.org/. Accessed 10 Sept 2019.

37. https://www.nationalmssociety.org/For-Professionals/ Researchers/Resources-for-Researchers/Clinical-StudyMeasures/Functional-Systems-Scores-(Fss)-and-ExpandedDisab. Accessed 10 Sept 2019.

38. https://www.nationalmssociety.org/For-Professionals/ Researchers/Resources-for-Researchers/Clinical-StudyMeasures/Multiple-Sclerosis-Quality-of-Life-54-(Msqol-54). Accessed 10 Sept 2019.

39. https://www.nationalmssociety.org/For-Professionals/ Researchers/Resources-for-Researchers/Clinical-StudyMeasures/Multiple-Sclerosis-Quality-of-Life-Inventory-(Msql). Accessed 10 Sept 2019.

40. https://www.psycharchives.org/Handle/20.500.12034/353. Accessed 10 Sept 2019.

41. Kaisey M, Solomon AJ, Luu M, Giesser BS, Sicotte NL. Incidence of multiple sclerosis misdiagnosis in referrals to two academic centers. Mult Scler Relat Disord. 2019;30:51-6.

42. Kalincik T, Cutter G, Spelman T, Jokubaitis V, Havrdova E, Horakova D, et al. Defining reliable disability outcomes in multiple sclerosis. Brain. 2015;138:3287-98.

43. Khalil M, Teunissen CE, Otto M, Piehl F, Sormani MP, Gattringer $\mathrm{T}$, et al. Neurofilaments as biomarkers in neurological disorders. Nat Rev Neurol. 2018;14:577-89.

44. Kleiter I, Gahlen A, Borisow N, et al. Apheresis therapies for NMOSD attacks: A retrospective study of 207 therapeutic interventions. Neurol Neuroimmunol Neuroinflamm. 2018;5:e504.

45. Koduah P, Paul F, Dorr JM. Vitamin D in the prevention, prediction and treatment of neurodegenerative and neuroinflammatory diseases. EPMA J. 2017;8:313-25.

46. Krupp LB, Larocca NG, Muir-Nash J, et al. The fatigue severity scale. Application to patients with multiple sclerosis and systemic lupus erythematosus. Arch Neurol. 1989;46:1121-3.

47. Kuchling J, Brandt AU, Paul F, Scheel M. Diffusion tensor imaging for multilevel assessment of the visual pathway: possibilities for personalized outcome prediction in autoimmune disorders of the central nervous system. EPMA J. 2017;8:279-94. 
48. Langdon DW, Amato MP, Boringa J, et al. Recommendations for a Brief International Cognitive Assessment for Multiple Sclerosis (BICAMS). Mult Scler. 2012;18:891-8.

49. Leppert D, Kuhle J. Blood neurofilament light chain at the doorstep of clinical application. Neurol Neuroimmunol Neuroinflamm. 2019;6:e599.

50. Martinez-Lapiscina EH, Arnow S, Wilson JA, Saidha S, Preiningerova JL, Oberwahrenbrock T, et al. Retinal thickness measured with optical coherence tomography and risk of disability worsening in multiple sclerosis: a cohort study. Lancet Neurol. 2016;15:574-84.

51. Miller DM, Bethoux F, Victorson D, et al. Validating Neuro-QoL short forms and targeted scales with people who have multiple sclerosis. Mult Scler. 2016;22:830-41.

52. Oberwahrenbrock T, Traber GL, Lukas S, et al. Multicenter reliability of semiautomatic retinal layer segmentation using OCT. Neurol Neuroimmunol Neuroinflamm. 2018;5:e449.

53. Oertel FC, Zimmermann H, Paul F, Brandt AU. Optical coherence tomography in neuromyelitis optica spectrum disorders: potential advantages for individualized monitoring of progression and therapy. EPMA J. 2018;9:21-33.

54. Oertel FC, Zimmermann HG, Brandt AU, et al. Novel uses of retinal imaging with optical coherence tomography in multiple sclerosis. Expert Rev Neurother. 2018:1-13.

55. Ontaneda D, Fox RJ, Chataway J. Clinical trials in progressive multiple sclerosis: lessons learned and future perspectives. Lancet Neurol. 2015;14:208-23.

56. Pasquier B, Borisow N, Rasche L, et al. Quantitative 7T MRI does not detect occult brain damage in neuromyelitis optica. Neurol Neuroimmunol Neuroinflamm. 2019;6:e541.

57. Paul F. Pathology and MRI: exploring cognitive impairment in MS. Acta Neurol Scand. 2016;134(Suppl 200):24-33.

58. Paul F, Ruprecht K. Current immunotherapy of multiple sclerosis. Nervenarzt. 2015;86:1031-42 quiz 1043-1034.

59. Pawlitzki M, Neumann J, Kaufmann J, et al. Loss of corticospinal tract integrity in early MS disease stages. Neurol Neuroimmunol Neuroinflamm. 2017;4:e399.

60. Penner IK, Paul F. Fatigue as a symptom or comorbidity of neurological diseases. Nat Rev Neurol. 2017;13:662-75.

61. Radue EW, Sprenger T, Gaetano L, et al. Teriflunomide slows BVL in relapsing MS: A reanalysis of the TEMSO MRI data set using SIENA. Neurol Neuroimmunol Neuroinflamm. 2017;4: e390.

62. Rahn AC, Kopke S, Stellmann JP, et al. Magnetic resonance imaging as a prognostic disability marker in clinically isolated syndrome: A systematic review. Acta Neurol Scand. 2019;139:18-32.

63. Reen GK, Silber E, Langdon DW. Multiple sclerosis patients understanding and preferences for risks and benefits of diseasemodifying drugs: A systematic review. J Neurol Sci. 2017;375: $107-22$.

64. Rissanen E, Tuisku J, Vahlberg T, et al. Microglial activation, white matter tract damage, and disability in MS. Neurol Neuroimmunol Neuroinflamm. 2018;5:e443.

65. Rothwell PM, Mcdowell Z, Wong CK, et al. Doctors and patients don't agree: cross sectional study of patients' and doctors' perceptions and assessments of disability in multiple sclerosis. BMJ. 1997;314:1580-3.

66. Schaffler N, Schonberg P, Stephan J, et al. Comparison of patientreported outcome measures in multiple sclerosis. Acta Neurol Scand. 2013;128:114-21.

67. Schlemm L, Chien C, Bellmann-Strobl J, et al. Gadopentetate but not gadobutrol accumulates in the dentate nucleus of multiple sclerosis patients. Mult Scler. 2017;23:963-72.

68. Sepulveda M, Delgado-Garcia G, Blanco Y et al. Late-onset neuromyelitis optica spectrum disorder: The importance of autoantibody serostatus. Neurol Neuroimmunol Neuroinflamm. 2019. https://doi.org/10.1212/NXI.0000000000000607.

69. Sinnecker T, Kuchling J, Dusek P, et al. Ultrahigh field MRI in clinical neuroimmunology: a potential contribution to improved diagnostics and personalised disease management. EPMA J. 2015;6:16

70. Sinnecker T, Schumacher S, Mueller K, et al. MRI phase changes in multiple sclerosis vs neuromyelitis optica lesions at 7T. Neurol Neuroimmunol Neuroinflamm. 2016;3:e259.

71. Solomon AJ, Corboy JR. The tension between early diagnosis and misdiagnosis of multiple sclerosis. Nat Rev Neurol. 2017;13:56772.

72. Spano B, Giulietti G, Pisani V, et al. Disruption of neurite morphology parallels MS progression. Neurol Neuroimmunol Neuroinflamm. 2018;5:e502.

73. Thebault S, Tessier DR, Lee H, Bowman M, Bar-Or A, Arnold DL, et al. High serum neurofilament light chain normalizes after hematopoietic stem cell transplantation for MS. Neurol Neuroimmunol Neuroinflamm. 2019;6:e598.

74. Torkildsen O, Myhr KM, Bo L. Disease-modifying treatments for multiple sclerosis - a review of approved medications. Eur J Neurol. 2016;23(Suppl 1):18-27.

75. Van Schependom J, D'hooghe MB, Cleynhens K, et al. The Symbol Digit Modalities Test as sentinel test for cognitive impairment in multiple sclerosis. Eur J Neurol. 2014;21(1219-1225): e1271-12.

76. Varhaug KN, Barro C, Bjornevik K, et al. Neurofilament light chain predicts disease activity in relapsing-remitting MS. Neurol Neuroimmunol Neuroinflamm. 2018;5:e422.

77. Veauthier C, Paul F. Sleep disorders in multiple sclerosis and their relationship to fatigue. Sleep Med. 2014;15:5-14.

78. Veauthier C, Gaede G, Radbruch H, Wernecke KD, Paul F. Sleep Disorders Reduce Health-Related Quality of Life in Multiple Sclerosis (Nottingham Health Profile Data in Patients with Multiple Sclerosis). Int J Mol Sci. 2015;16:16514-28.

79. Veauthier C, Hasselmann H, Gold SM, et al. The Berlin Treatment Algorithm: recommendations for tailored innovative therapeutic strategies for multiple sclerosis-related fatigue. EPMA J. 2016;7: 25.

80. Von Bismarck O, Dankowski T, Ambrosius B, et al. Treatment choices and neuropsychological symptoms of a large cohort of early MS. Neurol Neuroimmunol Neuroinflamm. 2018;5:e446.

81. Wang C, Barnett MH, Yiannikas C, et al. Lesion activity and chronic demyelination are the major determinants of brain atrophy in MS. Neurol Neuroimmunol Neuroinflamm. 2019. https://doi. org/10.1212/NXI.0000000000000593.

82. Weideman AM, Tapia-Maltos MA, Johnson K, et al. Metaanalysis of the Age-Dependent Efficacy of Multiple Sclerosis Treatments. Front Neurol. 2017;8:577.

83. Zeydan B, Gu X, Atkinson EJ, et al. Cervical spinal cord atrophy: An early marker of progressive MS onset. Neurol Neuroimmunol Neuroinflamm. 2018;5:e435.

84. Zimmermann HG, Knier B, Oberwahrenbrock T, Behrens J, Pfuh C, Aly L, et al. Association of Retinal Ganglion Cell Layer Thickness With Future Disease Activity in Patients With Clinically Isolated Syndrome. JAMA Neurol. 2018;75:1071-9.

\section{Further References (only to Table1: Published pivotal phase III trials)}

1. Torkildsen O, Myhr KM, Bo L. Disease-modifying treatments for multiple sclerosis - a review of approved medications. Eur J Neurol. 2016;23(Suppl 1):18-27. 
2. Interferon beta-1b is effective in relapsing-remitting multiple sclerosis. I. Clinical results of a multicenter, randomized, double-blind, placebo-controlled trial. The IFNB Multiple Sclerosis Study Group. Neurology. 1993;43:655-61.

3. O'Connor P, Filippi M, Arnason B, Comi G, Cook S, Goodin D, et al. 250 microg or 500 microg interferon beta- $1 \mathrm{~b}$ versus $20 \mathrm{mg}$ glatiramer acetate in relapsing-remitting multiple sclerosis: a prospective, randomised, multicentre study. Lancet Neurol. 2009;8: 889-97.

4. Durelli L, Verdun E, Barbero P, Bergui M, Versino E, Ghezzi A, et al. Every-other-day interferon beta-1b versus once-weekly interferon beta-1a for multiple sclerosis: results of a 2-year prospective randomised multicentre study (INCOMIN). Lancet. 2002;359: 1453-60.

5. Freedman MS. Evidence for the efficacy of interferon beta- $1 \mathrm{~b}$ in delaying the onset of clinically definite multiple sclerosis in individuals with clinically isolated syndrome. Ther Adv Neurol Disord. 2014;7:279-88.

6. Jacobs LD, Cookfair DL, Rudick RA, Herndon RM, Richert JR, Salazar AM, et al. Intramuscular interferon beta-1a for disease progression in relapsing multiple sclerosis. The Multiple Sclerosis Collaborative Research Group (MSCRG). Ann Neurol. 1996;39: 285-94.

7. Randomised double-blind placebo-controlled study of interferon beta-1a in relapsing/remitting multiple sclerosis. PRISMS (Prevention of Relapses and Disability by Interferon beta-1a Subcutaneously in Multiple Sclerosis) Study Group. Lancet. 1998;352:1498-504.

8. Mikol DD, Barkhof F, Chang P, Coyle PK, Jeffery DR, Schwid SR, et al. Comparison of subcutaneous interferon beta-1a with glatiramer acetate in patients with relapsing multiple sclerosis (the REbif vs Glatiramer Acetate in Relapsing MS Disease [REGARD] study): a multicentre, randomised, parallel, open-label trial. Lancet Neurol. 2008;7:903-14.

9. Panitch H, Goodin DS, Francis G, Chang P, Coyle PK, O'Connor P, et al. Randomized, comparative study of interferon beta-1a treatment regimens in MS: The EVIDENCE Trial. Neurology. 2002;59: 1496-506.

10. Johnson KP, Brooks BR, Cohen JA, Ford CC, Goldstein J, Lisak $\mathrm{RP}$, et al. Copolymer 1 reduces relapse rate and improves disability in relapsing-remitting multiple sclerosis: results of a phase III multicenter, double-blind placebo-controlled trial. The Copolymer 1 Multiple Sclerosis Study Group. Neurology. 1995;45:1268-76.

11. Comi G, Martinelli V, Rodegher M, et al. Effect of glatiramer acetate on conversion to clinically definite multiple sclerosis in patients with clinically isolated syndrome (PreCISe study): a randomised, double-blind, placebo-controlled trial. Lancet. 2009;374:1503-11.

12. Hartung HP, Gonsette R, Konig N, et al. Mitoxantrone in progressive multiple sclerosis: a placebo-controlled, double-blind, randomised, multicentre trial. Lancet. 2002;360:2018-25.

13. Polman CH, O'Connor PW, Havrdova E, Hutchinson M, Kappos $\mathrm{L}$, Miller DH, et al. A randomized, placebo-controlled trial of natalizumab for relapsing multiple sclerosis. N Engl J Med. 2006;354:899-910.

14. Kappos L, Radue EW, O'Connor P, Polman C, Hohlfeld R, Calabresi $\mathrm{P}$, et al. A placebo-controlled trial of oral fingolimod in relapsing multiple sclerosis. N Engl J Med. 2010;362:387-401.

15. Calabresi PA, Radue EW, Goodin D, Jeffery D, Rammohan KW, Reder AT, et al. Safety and efficacy of fingolimod in patients with relapsing-remitting multiple sclerosis (FREEDOMS II): a double- blind, randomised, placebo-controlled, phase 3 trial. Lancet Neurol. 2014;13:545-56.

16. Cohen JA, Barkhof F, Comi G, et al. Oral fingolimod or intramuscular interferon for relapsing multiple sclerosis. N Engl J Med. 2010;362:402-15.

17. Confavreux C, O'Connor P, Comi G, Freedman MS, Miller AE, Olsson TP, et al. Oral teriflunomide for patients with relapsing multiple sclerosis (TOWER): a randomised, double-blind, placebo-controlled, phase 3 trial. Lancet Neurol. 2014;13:247-56.

18. O'Connor P, Wolinsky JS, Confavreux C, Comi G, Kappos $\mathrm{L}$, Olsson TP, et al. Randomized trial of oral teriflunomide for relapsing multiple sclerosis. N Engl J Med. 2011;365: 1293-303.

19. Vermersch P, Czlonkowska A, Grimaldi LM, Confavreux C, Comi G, Kappos L, et al. Teriflunomide versus subcutaneous interferon beta-1a in patients with relapsing multiple sclerosis: a randomised, controlled phase 3 trial. Mult Scler. 2014;20:705-16.

20. Cohen JA, Coles AJ, Arnold DL, Confavreux C, Fox EJ, Hartung $\mathrm{HP}$, et al. Alemtuzumab versus interferon beta $1 \mathrm{a}$ as first-line treatment for patients with relapsing-remitting multiple sclerosis: a randomised controlled phase 3 trial. Lancet. 2012;380:1819-28.

21. Coles AJ, Twyman CL, Arnold DL, Cohen JA, Confavreux C, Fox EJ, et al. Alemtuzumab for patients with relapsing multiple sclerosis after disease-modifying therapy: a randomised controlled phase 3 trial. Lancet. 2012;380:1829-39.

22. Gold R, Kappos L, Arnold DL, Bar-Or A, Giovannoni G, Selmaj K, et al. Placebo-controlled phase 3 study of oral BG-12 for relapsing multiple sclerosis. N Engl J Med. 2012;367:1098-107.

23. Fox RJ, Miller DH, Phillips JT, Hutchinson M, Havrdova E, Kita $\mathrm{M}$, et al. Placebo-controlled phase 3 study of oral BG-12 or glatiramer in multiple sclerosis. N Engl J Med. 2012;367:1087-97.

24. Calabresi PA, Kieseier BC, Arnold DL, Balcer LJ, Boyko A, Pelletier $\mathrm{J}$, et al. Pegylated interferon beta-1a for relapsingremitting multiple sclerosis (ADVANCE): a randomised, phase 3, double-blind study. Lancet Neurol. 2014;13:657-65.

25. Kappos L, Wiendl H, Selmaj K, Arnold DL, Havrdova E, Boyko A, et al. Daclizumab HYP versus Interferon Beta-1a in Relapsing Multiple Sclerosis. N Engl J Med. 2015;373:1418-28.

26. Liu Y, Vollmer T, Havrdova E, Riester K, Lee A, Phillips G, et al. Impact of daclizumab versus interferon beta-1a on patient-reported outcomes in relapsing-remitting multiple sclerosis. Mult Scler Relat Disord. 2017;11:18-24.

27. Giovannoni G, Comi G, Cook S, Rammohan K, Rieckmann P, Soelberg Sørensen P, et al. A placebo-controlled trial of oral cladribine for relapsing multiple sclerosis. N Engl J Med. 2010;362:416-26.

28. Giovannoni G, Soelberg Sorensen P, Cook S, et al. Safety and efficacy of cladribine tablets in patients with relapsing-remitting multiple sclerosis: Results from the randomized extension trial of the CLARITY study. Mult Scler. 2018;24:1594-604.

29. Hauser SL, Bar-Or A, Comi G, Giovannoni G, Hartung HP, Hemmer B, et al. Ocrelizumab versus Interferon Beta-1a in Relapsing Multiple Sclerosis. N Engl J Med. 2017;376:221-34.

30. Montalban X, Hauser SL, Kappos L, Arnold DL, Bar-Or A, Comi $\mathrm{G}$, et al. Ocrelizumab versus Placebo in Primary Progressive Multiple Sclerosis. N Engl J Med. 2017;376:209-20.

31. De Stefano N, Comi G, Kappos L, et al. Efficacy of subcutaneous interferon beta-1a on MRI outcomes in a randomised controlled trial of patients with clinically isolated syndromes. J Neurol Neurosurg Psychiatry. 2014;85:647-53.

32. Comi G, De Stefano N, Freedman MS, et al. Subcutaneous interferon beta-1a in the treatment of clinically isolated syndromes: 3 year and 5-year results of the phase III dosing frequency-blind 
multicentre REFLEXION study. J Neurol Neurosurg Psychiatry. 2017;88:285-94.

33. Jacobs LD, Beck RW, Simon JH, Kinkel RP, Brownscheidle CM, Murray TJ, et al. Intramuscular interferon beta-1a therapy initiated during a first demyelinating event in multiple sclerosis. CHAMPS Study Group. N Engl J Med. 2000;343:898-904.

34. Comi G, De Stefano N, Freedman MS, et al. Comparison of two dosing frequencies of subcutaneous interferon beta-1a in patients with a first clinical demyelinating event suggestive of multiple sclerosis (REFLEX): a phase 3 randomised controlled trial. Lancet Neurol. 2012;11:33-41.

35. https://www.iqwig.de/download/A15-12_Fingolimod Nutzenbewertung-35a-SGB-V.pdf

36. https://www.iqwig.de/download/A13-38_Teriflunomid_ Nutzenbewertung-35a-SGB-V.pdf

Publisher's note Springer Nature remains neutral with regard to jurisdictional claims in published maps and institutional affiliations. 